\title{
Genetic Mapping and Validation of Quantitative Trait Loci (QTL) for the Grain Appearance and Quality Traits in Rice (Oryza sativa L.) by Using Recombinant Inbred Line (RIL) Population
}

\author{
Leila Bazrkar-Khatibani $\mathbb{D}^{1},{ }^{1}$ Barat-Ali Fakheri, ${ }^{1}$ Maryam Hosseini-Chaleshtori, ${ }^{2}$ \\ Anumalla Mahender ${ }^{D},{ }^{3}$ Nafiseh Mahdinejad, ${ }^{1}$ and Jauhar Ali ${ }^{3}{ }^{3}$ \\ ${ }^{1}$ Plant Breeding and Biotechnology Department, Zabol University, Iran \\ ${ }^{2}$ Rice Research Institute of Iran (RRII), Agricultural Research, Education and Extension Organization, Rasht 1658, Iran \\ ${ }^{3}$ Rice Breeding Platform, International Rice Research Institute (IRRI), Los Baños, Laguna 4031, Philippines \\ Correspondence should be addressed to Jauhar Ali; J.Ali@irri.org
}

Received 3 September 2018; Revised 16 January 2019; Accepted 22 January 2019; Published 25 February 2019

Academic Editor: Atsushi Kurabayashi

Copyright (C) 2019 Leila Bazrkar-Khatibani et al. This is an open access article distributed under the Creative Commons Attribution License, which permits unrestricted use, distribution, and reproduction in any medium, provided the original work is properly cited.

\begin{abstract}
Rice grain shape and nutritional quality traits have high economic value for commercial production of rice and largely determine the market price, besides influencing the global food demand for high-quality rice. In order to understand the genetic components of grain appearance traits in paddy, brown, and head rice, 15 traits were evaluated by using 157 recombinant inbred lines (RILs) derived from a cross between two Iranian rice cultivars Ali-Kazemi (A) and Kadous (K). A significant variation was observed and showed transgressive segregation among the RILs. Correlations between the visual appearances of grain traits were studied. A linkage map with 65 polymorphic SSR markers was constructed, which covered $1517.32 \mathrm{cM}$ of the rice genome. A total of seven QTLs were identified on four chromosomes, 1, 6, 9, and 12, associated with four traits, which are explained by the total phenotypic variation of $44.27 \%$ and LOD score of 32.77 in 2014 and 2015, respectively. Among these, four QTLs for two traits were consistently flanked by RM23904 and RM24432 on chromosome 9. Single QTL for head grain length (HGL) expressed in both the years on chromosomes 1 and 9. A major QTL for seed weight was detected on chromosome 9, which explained $10.18 \%$ of the phenotypic variation. The additive effect of all the QTLs was positively contributed by Ali-Kazemi allele, except one QTL on chromosome 6 ( $\left.q B G L_{-} 6\right)$ that showed a negative additive effect being contributed by the Kadous allele. The study also validated the identified QTLs with the polymorphic SSR markers that were previously reported. Novel QTLs were identified on chromosomes 6 and 9, and many of the polymorphic markers were found to be associated with milling processing of grain quality, cooking, and nutraceutical properties of rice by extensive literature and database analysis. Therefore, these validated QTLs and marker information could be utilized in the marker-assisted selection to improve grain appearance and nutritional grain quality traits in rice.
\end{abstract}

\section{Introduction}

Rice (Oryza sativa L.), is one of the major staple food crops for more than 3.5 billion global population. The production and consumption of global rice accounted for almost $90 \%$ by Asian countries; mainly China and India alone contribute about $55 \%$ [1]. Among the cereals, rice provides up to $20 \%$ of their regular calorie intake for millions of global population. In order to ensure nutritional food security, the projected rice production must be increased to 852 million tons by 2035 [2]. With the intensifications of diverse food demands and living standards of global populations, rice grain appearance and quality have become a primary concern for rice breeders. Therefore, there is an urgent need to increase the grain yield along with desirable grain nutritional quality (GQ) traits in rice $[3,4]$. The rice grain attributed traits are a major contributor to the determination of the acceptability by end users and market price $[5,6]$. GQ has been recognized into two 
classes: (i) grain appearance and (ii) cooking and eating qualities [7]. The grain appearance determined by the length, breadth, length-breadth ratio, endosperm translucency, and cooking and eating quality traits includes aroma, volume expansion, cooked kernel elongation, firmness/stickiness (related to amylose content), gelatinization temperature (measured as the alkali spreading value), and mouthfeel $[8,9]$. The GQ traits are known to be associated with physical appearance of grain size with several aspects of grain quality attributes related to cooking, nutritional, and sensory characteristics $[10,11]$.

Globally, the rice producers' and consumers' major concern is grain appearance which also contributes to the reputation of a given variety. Although the preferences for rice grain characteristics vary with different consumer groups, long and slender rice is usually preferred by many consumers in India, Vietnam, USA, and most of the Asian countries [7, 12], whereas short and round rice grain is consumed in Northern China, Korea, and Japan. The long-grained rice varieties tend to produce dry, fluffy, and separated grains after being cooked, whereas the medium and short grain varieties tend to produce clumped, moist, and chewy grains after cooking [13]. The GQ trait inheritance in rice is complex that includes many components. Among them, amylose content (AC), gelatinization temperature (GT), gel consistency (GC), and protein content (PC) have been extensively studied and found to be controlled by QTLs with major and minor effects [9].

The milling yield or milling efficiency is another major factor to determine the percentage of the whole kernel milled rice obtained from rough rice (paddy rice) after milling, and the price of whole grain is typically twice as that of broken grains [14]. Therefore, it is an economically important trait. During milling processes, it combines multiple steps such as hulling and removal of the bran and embryo, followed by separating whole grains from broken grains. This gives rise to three milling components: brown rice ratio, milled rice ratio, and head rice ratio [15]. Another component of this process yields rice bran, an essential source of protein, vitamins, minerals, antioxidants, and phytosterols [16]. Rice bran protein has immense potential in the food industry, having unique nutraceutical properties and reported as a hypoallergenic food ingredient in infant formulations and anticancer properties [17].

With the increase in literacy percentage and awareness about diets, the people showed a tendency to be more health conscious and interested to take nutritionally enriched food. The quality of rice is an important character to determine the economic value in the export market and consumer acceptance [11]. The preference for rice quality varies across regions, as Japanese people prefer short grain and sticky rice [18]. Conversely, the whole northern part of India, Pakistan, and the Middle East regions prefer Basmati rice. The appearance of GQ has been determined by grain length, width, the length-width ratio (LWR), chalkiness of the endosperm, and grain shape, and size has a direct effect on the marketability and consumer acceptance [19]. The rice consumers' preference for grain shape differs from one location to another. Therefore, grain shape trait needs to be understood and considered in the context of market preference of the region and breeding approaches. The physical appearance of rice grain such as the shape and width greatly influences other vital rice quality traits, viz., endosperm chalkiness, milling efficiency, and cooking and eating properties [20]. Identification and development of superior GQ traits by direct examination in the field are very difficult due to trait complexity and being easily influenced by the environment $[21,22]$. Therefore, with the recent development of DNA molecular marker technology and physical linkage map of rice, it has become feasible to dissect complex polygenic traits in rice. Thus, there is a need to understand the genetic basis of GQ traits and develop suitable strategies to develop the consumer preferred type of quality rice.

In a corollary of classical plant breeding methods, with the intervention of molecular markers and advanced genomic techniques, existing varieties could be improved to target the most vulnerable people of developing nations from micronutrient deficiency $[9,10]$. The major targets to enhance rice GQ include appearance, milling, cooking, and physicochemical properties and nutritional qualities in rice. Many of these traits are highly complex governed by quantitative trait loci (QTLs) that are influenced by environmental factors and showed genetic variation to be polygenic in segregating progeny of intervarietal crosses, natural populations as landraces, and among inbred lines. The efficiency of molecular markers for the improvement of grain appearance and nutritional quality traits has been successfully utilized in various genetic backgrounds, mapping populations, and landraces of rice genotypes [10, 23-27]. Among the various types of molecular markers, SSR, STS, and SNP markers are highly useful when they are located within a gene of interest or in linkage disequilibrium with the gene throughout a population.

In the last several years, many QTLs for grain appearance and eating quality have been identified from different mapping populations: RILs, DHs, CSSLs, $\mathrm{F}_{2}$, and $\mathrm{BC}_{2} \mathrm{~F}_{2}$ (reviewed by Mahender et al. [9]). Over the past two decades, many QTLs associated with grain dimension have been mapped in several populations of different chromosomes in rice. From the comprehensive literature survey and Gramene database, we found a significant number of QTLs distributed on different chromosomes. A total of 59 QTLs for MPGQ, 71 QTLs for GA, 36 QTLs for CP, seven QTLs for FRG, and 86 QTLs for NF were identified to date (Supplementary Table 1). Another critical trait is grain chalkiness (GCH), which influences the grain quality and market price. About 82 QTLs related with GCH traits, including 30 QTLs for percentage grain with white core (PGWC), 26 QTLs for degree of endosperm chalkiness (DEC), 12 QTLs for area of endosperm chalkiness (AEC), 11 QTLs for white-backed kernel, and 3 QTLs for basal white (BW), were reported $[28,29]$ in the Gramene database. Among them, the majority of the QTLs with $\geq 3$ LOD score were located on chromosome $1[5,30]$, chromosome 2 [30, 31], chromosome $3[21,32-34]$, chromosome $4[35,36]$, chromosome $5[37,38]$, chromosome $6[32,36]$, chromosome 7 [5, 30, 38, 39], and chromosome 10 [37]. $\mathrm{Up}$ to date, a total of 14 major genes were significantly 
involved in controlling the grain shape and size, totally, four genes (GW2, GW8, GS5, and GW5/qSW5) for grain width, eight genes (GS2/GL2, GL3.1/qGL3, GL4, GLW7, GS3, OsLG3, OsLG3b/qLGY3, and TGW6) for grain length, and two genes (GW6a and GL7/GW7) for similar effects on grain length, width, and also in larger impact on grain weight $[40,41]$. Despite these QTL mapping efforts, only limited information is available for a combination of the physical appearance of paddy, brown, and head rice grain visual quality traits and associated QTL information. Based on the comprehensive information of grain appearance and quality traits, associated QTLs, in the present study, were investigated by using recombinant inbred lines (RILs) derived from a cross between indica species of two Iranian rice cultivars such as Ali-Kazemi which is a native rice cultivar from Guilan Province of northern Iran and improved rice cultivar of Kadous. Interestingly, in more than $60 \%$ of rice-growing areas in Iran, farmers have been using many traditional and local rice varieties for their primary concern to grain nutritional and cooking quality. However, the genetic analyses of this background of parents and RILs have not been explored. Therefore, the present study was carried out by considering the following objectives: (i) to evaluate grain appearance traits and their correlations, (ii) to identify the QTLs by using composite interval mapping method, and (iii) to validate the QTL-linked molecular markers with previously reported QTLs.

\section{Materials and Methods}

2.1. Plant Materials. Parental lines, Oryza sativa ssp. indica cv. Ali-Kazemi which is a local rice cultivar and Kadous which is an improved Iranian rice variety, were used for the development of mapping population to identify genomic region conferring grain appearance traits. The native rice cultivar of Ali-Kazemi is adapted to the northern region of Iran, and it possesses better grain and cooking quality traits (grain yield: $3.8-4$ tha $^{-1}$; amylose content: 16.7; gelatinization temperature: 4.9; gel consistency: 70 ; the average length of grain before cooking: $7.2 \mathrm{~mm}$, after cooking: $11.6 \mathrm{~mm}$; width 2.1-2.3 $\mathrm{mm}$; and the elongation ratio: 1.6). The improved rice cultivar of Kadous provides high grain yield and their grain quality traits as grain yield: $6.5-7 \mathrm{tha}^{-1}$; amylose content: 23.25; gelatinization temperature: 3.3 ; gel consistency: 52 ; the average length of grain before cooking: $7.8 \mathrm{~mm}$, after cooking: $10.25 \mathrm{~mm}$; width $\leq 2 \mathrm{~mm}$; and the elongation ratio: 1.3 [42]. This improved rice variety was developed from two International Rice Research Institute (IRRI) lines IR67015-94-2-3 and IR64669-153-2-3, respectively. A total of 157 RILs in $F_{9}$ generation were developed by using single seed descent (SSD) [43]. The RILs and parents were planted during the rice-growing seasons of 2014 and 2015 in the experimental farms of the Rice Research Institute of Iran (RRII) located in the northwestern area of Rasht in Guilan, Iran. The $\mathrm{F}_{9}$ plants and parents were raised in a randomized complete block design with three replications with a spacing of $25 \times 25 \mathrm{~cm}$. Field's management followed the normal agricultural practices.
2.2. Phenotyping. Mature $\mathrm{F}_{9}$ grains of the RILs and parental lines were air-dried and stored at room temperature $\left(37-40^{\circ} \mathrm{C}\right)$ for at least two months before milling. The moisture content of each sample was reduced to $14 \%$ for both years. The length and width of white rice were measured by a photoenlarger, and the length, width, and thickness of paddy and brown rice were measured using digital Vernier calipers $(0-150 \mathrm{~mm})$. Meanwhile, the length-to-width ratios were estimated using the obtained data. The percentage of chalkiness was also determined by counting the number of chalky grains selected from 100 grains for each line. A total of 15 grain appearance traits in paddy, brown, and head rice were followed by paddy grain length (PGL), paddy grain width (PGW), paddy grain thickness (PGT), paddy grain length-to-width ratio (PGLWR), paddy grain length-to-thickness ratio (PGLTR), brown grain length (BGL), brown grain width (BGW), brown grain thickness (BGT), brown grain length-to-width ratio (BGLWR), brown grain length-to-thickness ratio (BGLTR), head grain length (HGL), head grain width (HGW), head grain length-to-width ratio (HGLWR), seed weight (SW), and chalkiness $(\mathrm{CH})$ and were recorded. Further, phenotypic trait investigation was followed by descriptive statistical analysis which are the mean, range, standard deviation, the coefficient of variation, skewness, and kurtosis information which were calculated by using STAR software tool version 1.4 package (http://bbi.irri.org) and MS Excel. Also, the Pearson correlation coefficient was estimated between the phenotypic traits by using the corrplot package in R software (https://github.com/taiyun/corrplot).

2.3. Genotyping by SSR Markers. Plant genomic DNA was isolated from fresh and young leaf tissue for each RIL and the parents following cetyltrimethyl-ammonium bromide (CTAB) method slightly modified from that used by Murray and Thompson [44]. A total of 300 simple sequence repeat (SSR) markers, RM-series [45, 46], were utilized to survey polymorphism, of which 65 polymorphic markers were well distributed among 12 chromosomes (Table 1). Polymerase chain reaction (PCR) was performed in $10 \mu \mathrm{l}$ volume containing $2 \mu \mathrm{l}$ of template DNA ( $25 \mathrm{ng}), 0.4 \mu \mathrm{l}$ of forward and reverse primers each at 10 pmol concentration, $0.6 \mu \mathrm{l}$ dNTPs $(2 \mathrm{mM}), 0.12 \mu \mathrm{l} \mathrm{Taq}$ DNA polymerase $(5 \mathrm{U} / \mu \mathrm{l}), 0.48 \mu \mathrm{l} \mathrm{MgCl}_{2}$

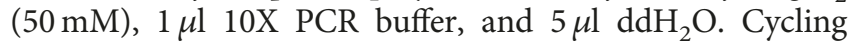
conditions followed in PCR were initial denaturation at $94^{\circ} \dot{\mathrm{C}}$ for $5 \mathrm{~min}$ followed by 35 cycles of denaturation at $94^{\circ} \mathrm{C}$ for $30 \mathrm{~s}$, primer annealing temperature for 30 s, extension at $50-58^{\circ} \mathrm{C}$ for $2 \mathrm{~min}$ and final extension at least $72^{\circ} \mathrm{C}$ for $5 \mathrm{~min}$. After PCR amplification, the PCR products were separated on $6 \%$ polyacrylamide gels (19:1-acrylamide:bisacrylamide) as described by Creste et al. [47] and Bassam and Caetano-Anollés [48] and visualized by silver staining.

2.4. Linkage Map Construction and QTL Analysis. Out of the 300 SSR markers, 65 polymorphic SSR markers were distributed throughout 12 chromosomes of rice. Genotypic data of 157 RILs and parents were transformed according to the requirement of IciMapping software v4.0 [49] to develop 


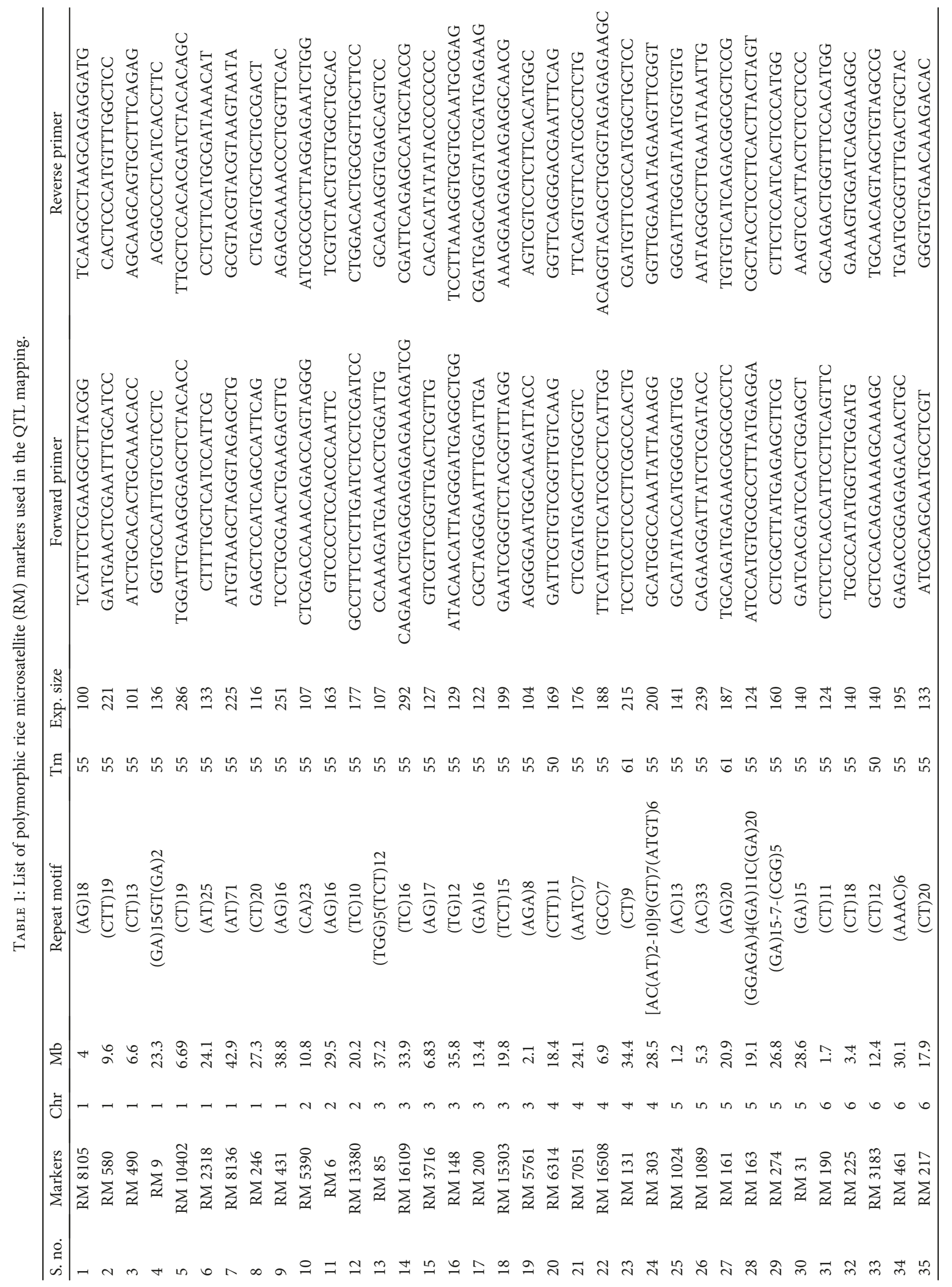




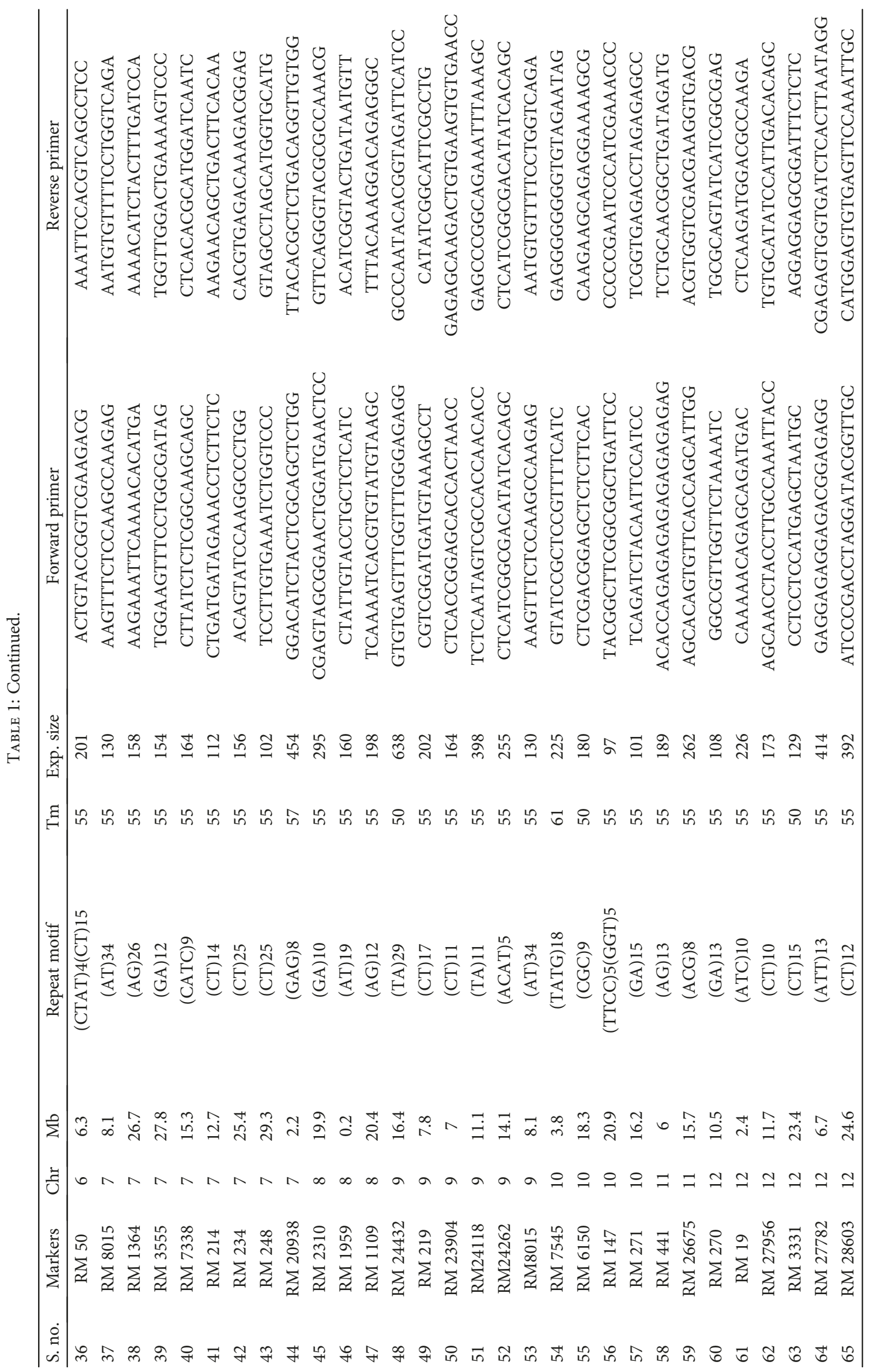



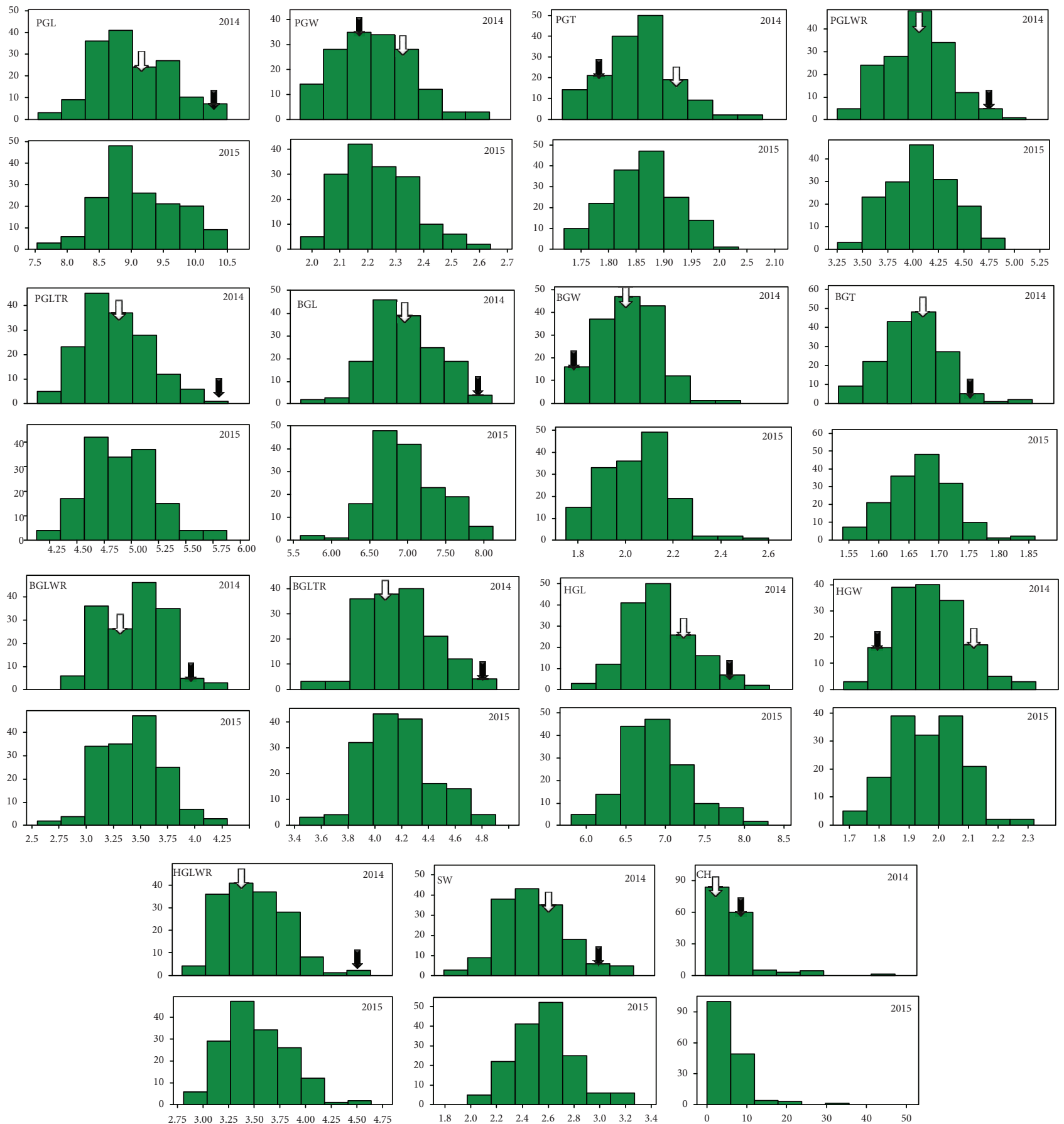

$\llbracket$ Ali-Kazemi

FIGURE 1: The histogram of frequency distribution of grain appearance quality traits under two seasons in rice.

the linkage map and also to identify the potential QTLs. The analysis of QTLs was carried out by using two statistical methods, namely, interval mapping (IM-ADD) and inclusive composite interval mapping (ICIM-ADD) methods [50] which were performed with a maximum recombination fraction of 0.3 and LOD threshold of 2.5 and 3.0 to claim the significant QTLs.

\section{Results}

3.1. Performance of Grain Appearance Traits for Parents and RIL Population. The recurrent and donor parental lines (Ali-Kazemi and Kadous) and RIL population of phenotypic traits are shown in Figure 1. The average values from Kadous are higher than those from Ali-Kazemi for all 15 traits except 

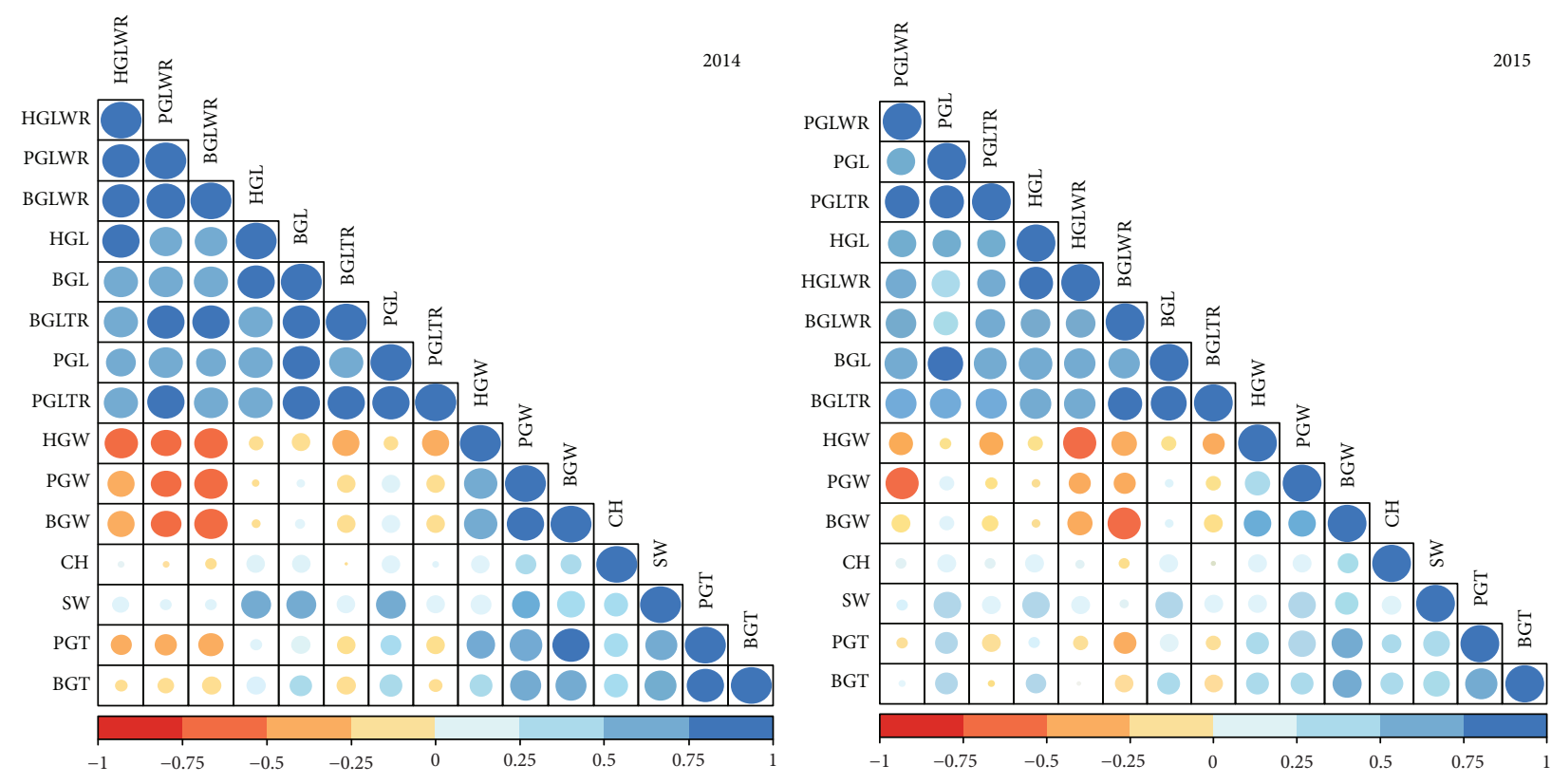

FiguRE 2: Correlation analysis of 15 traits of 157 RILs in 2014 and 2015.

PGL, BGW, BGT, and HGW in 2014, whereas PGW, PGT, BGW, BGT, HGW, and SW in 2015, respectively. All variables showed a positive asymmetry, varying between 0.59 (BGL) to $17.35(\mathrm{CH})$ in 2014 and 0.05 (PGLWR) to 13.16 $(\mathrm{CH})$ in 2015. Except for HGW (-0.28), the asymmetry shown was negative in 2015. The kurtosis values of four traits, PGW, BGW, BGLWR, and HGW, were negative in 2014. Similarly, in 2015, five traits, PGL, PGT, PGLWR, PGLTR, and SW, had a negative value, respectively. Therefore, the shape of the trait distribution pattern was classified as a platykurtic distribution. However, $\mathrm{SW}$ and $\mathrm{CH}$ had shown the highest coefficient variation among the RILs, whereas BGT showed the lowest CV value in both the years. Correlation analysis was carried out among the 15 traits in 2014 and 2015, and the results are represented in Figure 2. Many of the traits are positively significantly correlated with each other. The highest positive correlation was observed between PGL \& PGLTR $(r=0.86)$, PGW \& BGW $(r=0.94)$, PGLWR \& PGLTR $(r=0.85)$, PGLWR $\& \operatorname{BGLWR}(r=0.91)$, and BGL \& BGLTR $(r=0.88)$ in 2014, whereas the highest positive correlation was observed between PGL \& PGLTR $(r=0.86)$ and BGL \& BGLTR $(r=0.87)$ in 2015, respectively.

3.2. QTL Mapping for Grain Appearance Traits. Genetic mapping was performed by using all the 65 polymorphic SSR markers. A total of 65 RM-series markers were distributed across all chromosomes with a total coverage length of $1517.32 \mathrm{cM}$, of which chromosome 1 had the highest chromosomal spanning region of about $223.25 \mathrm{cM}$. A total of seven QTLs for grain appearance were identified on chromosomes 1, 6, 9, and 12 (Table 2; Figure 3), of which three QTLs from 2014 and four from 2015 were identified by ICIM-ADD methods. Each of these QTLs was explained by phenotypic variance (PV) which ranged from $4.02 \%$ to $10.18 \%$, except on chromosome 6 , which is associated with BGL and had shown negative additive effect of Kadous allele, whereas in the remaining, all the QTLs were contributed by the positive additive effect of Ali-Kazemi alleles.

In 2014, three QTLs ( $q H G L \_1, q C H \_9$, and $q C H \_12$ ) were distributed on chromosomes 1, 9, and 12. The QTLs were associated with two traits, $\mathrm{HGL}$ and $\mathrm{CH}$, which explained a total PV of $16.77 \%$, whereas in 2015, four QTLs ( $q B G L \_6, q H G L \_9, q S W \_9$, and $\left.q S W \_9-1\right)$ were identified on chromosomes 6 and 9, respectively. The head grain length QTL qHGL_1 is located on chromosome 1 at the $178 \mathrm{cM}$ position which were flanked by RM490, and RM2318 explained a total PV of $5.01 \%$. For grain chalkiness, two QTLs (qCH_9, and qCH_12) were detected in two chromosomes, 9 and 12, and explained a total PV of $11.76 \%$ and a LOD score of 11.05 , respectively. The region flanked by RM23904 and RM24432 n chromosome 9 consisting of four QTLs, qHGL_1, qHGL_9, qSW_9, and qSW_9-1, was responsible for the head grain length and seed weight, respectively. The head grain length QTLs were noticed on chromosomes 1 and 9 in both the years 2014 and 2015. Interestingly, none of them were overlapped QTLs across both the years in the chromosomal regions. Further, we validated the identified QTLs and also polymorphic markers with previously reported QTLs for grain appearance and quality traits in rice by comprehensive literature survey and the publicly accessible Gramene database (http://archive.gramene.org).

\section{Discussion}

The physical appearance of grain dimensions (length, breadth, and length/breadth ratio) is essential for grain quality traits in rice and highly preferred by consumers. The trait of grain shape is difficult for the plant breeders to improve the grain architecture appearance by conventional breeding methods [51, 52]. The use of the marker-assisted breeding and the completion of rice genome sequencing have greatly 
TABLE 2: Additive effects of QTLs for grain appearance detected by ICIM-ADD in two years.

\begin{tabular}{|c|c|c|c|c|c|c|c|c|}
\hline S. no. & QTL & Chr & Position (cM) & Flanking markers & $\mathrm{LOD}^{\mathrm{a}}$ & $\mathrm{PVE}^{\mathrm{b}}$ & Additive effect $^{c}$ & $\begin{array}{l}\text { The contribution of } \\
\text { the favorable allele }\end{array}$ \\
\hline 1 & $q H G L \_1(2014)$ & 1 & 178 & RM490-RM2318 & 10.26 & 5.01 & 10.57 & $\bar{A}$ \\
\hline 2 & qCH_9 (2014) & 9 & 77 & RM23904-RM24432 & 2.95 & 6.7 & 0.23 & A \\
\hline 3 & qCH_12 (2014) & 12 & 54 & RM270-RM27956 & 8.1 & 5.06 & 10.25 & A \\
\hline 4 & qBGL_6 (2015) & 6 & 52 & RM225-RM3183 & 3.01 & 4.02 & -0.07 & $\mathrm{~K}$ \\
\hline 5 & qHGL_9 (2015) & 9 & 77 & RM23904-RM24432 & 2.86 & 7.22 & 0.23 & A \\
\hline 6 & qSW_9(2015) & 9 & 78 & RM23904-RM24432 & 2.57 & 6.08 & 0.21 & A \\
\hline 7 & qSW_9-1 (2015) & 9 & 82 & RM23904-RM24432 & 3.02 & 10.18 & 0.11 & A \\
\hline
\end{tabular}

${ }^{a}$ Interval of the LOD peak value for QTL; ${ }^{b}$ interval of the LOD peak value for QTL; ${ }^{c}$ positive additive effect of QTL came from Ali-Kazemi, while negative effect came from Kadous.

facilitated the discovery and mapping of QTLs and genes for grain dimension. To date, several QTLs were reported for the dynamic shape of rice grain attributed traits in the various genetic background of mapping populations in rice $[9,10,27]$. However, different grain visualization traits regarding paddy, brown, and head rice grain appearance QTL information have not been reported. In this study, the focus was to dissect these traits using the RIL population, which were derived from a popular Iranian local rice cultivar of Ali-Kazemi (long-grain) and improved rice cultivar of Kadous, as well as to identify the possible DNA markers for selection of physical grain appearance traits. A total of seven QTLs were identified for four traits, HGL, BGL, SW, and CH, on the four chromosomes, 1, 6, 9, and 12, by using ICIM methods. The percentage of PV explained by each QTL was ranged from 4.02 to $10.18 \%$, with an average of $6.32 \%$. Among these seven QTLs, single QTL qSW_9-1 (2015) which accounted for PV was higher than 10\%, while the remaining six QTLs explained ranged from 4.02 to $7.22 \%$ (Table 2). The higher phenotypic variation explained for most of the studied traits revealed major genes/QTLs to be responsible. Out of the 15 traits, four traits were found to be governed by one or two QTLs that explained a significant PV. However, only QTLs for a single-trait HGL flanked by RM490-RM2318 on chromosome 1 and RM23904 and RM24432 on chromosome 9 were identified in both the years 2014 and 2015 . These QTLs are contributed by the positive additive effect of Ali-Kazemi allele.

4.1. Validation of the Identified QTLs and Polymorphic Markers and Their Significance. To date, several researchers had identified QTLs that were associated with physical appearance and grain quality traits and were located majorly on chromosomes $1,3,4,5,6,7$, and 10 [5, 10, 27, 36, 39]. In the present study, paddy, brown, and head grain rice appearance trait-related QTLs and polymorphic SSR markers were effectively compared with previously reported QTLs and linkages maps. The QTL intervals of RM490 to RM2318 on chromosome 1, RM225 to RM3183 on chromosome 6, RM23904 to RM24432 on chromosome 9, RM270 to RM27956 on chromosome 12, and also a few polymorphic SSR markers were associated with four different key component traits as follows: milling properties of grain quality
(MPGQ), grain appearance (GA), nutritional factors (NF), and cooking properties $(\mathrm{CP})$ in rice.

In our study, on chromosome 1, a single QTL $q H G L_{-} 1$ (2014) flanked by right SSR marker RM490 was significantly associated with three QTLs qmp1.1 [53], qCo.1 [36], and $q \mathrm{Fe} 1.2$ [54]. Also, we found that these QTLs were responsible for milling and mineral element content related to nutritional factors in grain quality. Interestingly, the polymorphic RM431 marker used in our study was associated with two QTLs related to head rice recovery (qhrr.1) and grain length (qgrl1-1). qhrr.1 identified by using $\mathrm{BC}_{2} \mathrm{~F}_{2}$ population was derived from a genetic background of IR64 and O. rufipogon mapping populations [55], whereas qgrl1-1 was reported by Amarawathi et al. [5] by using RILs from Pusa 1121 and Pusa 1342. Likewise, adjacent to the polymorphic marker RM8136 at the $42.7 \mathrm{Mb}$ region, $q B R R 1.2$ (brown rice rate) QTL was associated that accounted for $7.1 \%$ of PV [10].

$\mathrm{Lu}$ et al. [56] identified a total of 10 QTLs for five different mineral elements such as $\mathrm{Cu}, \mathrm{Ca}, \mathrm{Zn}, \mathrm{Mn}$, and Fe on six chromosomes 1, 2, 4, 5, 7, and 9, which explained a PV ranging from 5.3 to $25.81 \%$. Among these, two mineral element QTLs, $q C a .3$ and $q C u .1$, were similarly mapped on chromosome 3 which were associated with polymorphic marker RM200 used in our study at $13.4 \mathrm{Mb}$ and RM148 at $35.8 \mathrm{Mb}$ regions. Wang et al. [10] further detected that for the brown rice rate QTL, $q B R R 3$ was adjacent to RM16109 at $34.7 \mathrm{Mb}$ that accounted for $6.9 \%$ of PV. Similarly, RM131 at $34.4 \mathrm{Mb}$ and the adjacent RM6314 at the $18.2 \mathrm{Mb}$ region on chromosome 4 were controlling the amino acid content in rice grain ( $q A A .1)$ [57] and grain length (qGL4.1) [10]. The polymorphic marker used in our study has been found to be tightly linked to several important grain quality and nutrient traits. Such polymorphic markers could be fully utilized in rice breeding programs to mobilize the grain quality traits of interest in early generations.

Recently, Wang et al. [10] identified 72 QTLs for nine rice grain appearances, and milling quality traits were distributed on 12 chromosomes in two different environments by using a diverse panel of 258 rice accessions from the $3 \mathrm{~K}$ Rice Genome Project. Out of 72, 11 QTLs were overlapped or adjacent to the SSR marker positions on six different chromosomes, $1,3,4,5,7$, and 8 , in the current study. On chromosome 5, two QTLs qGLWR5 and $q P G W C 5$ associated with RM1089 at $5.3 \mathrm{Mb}$ regions and $q G W 5$ 


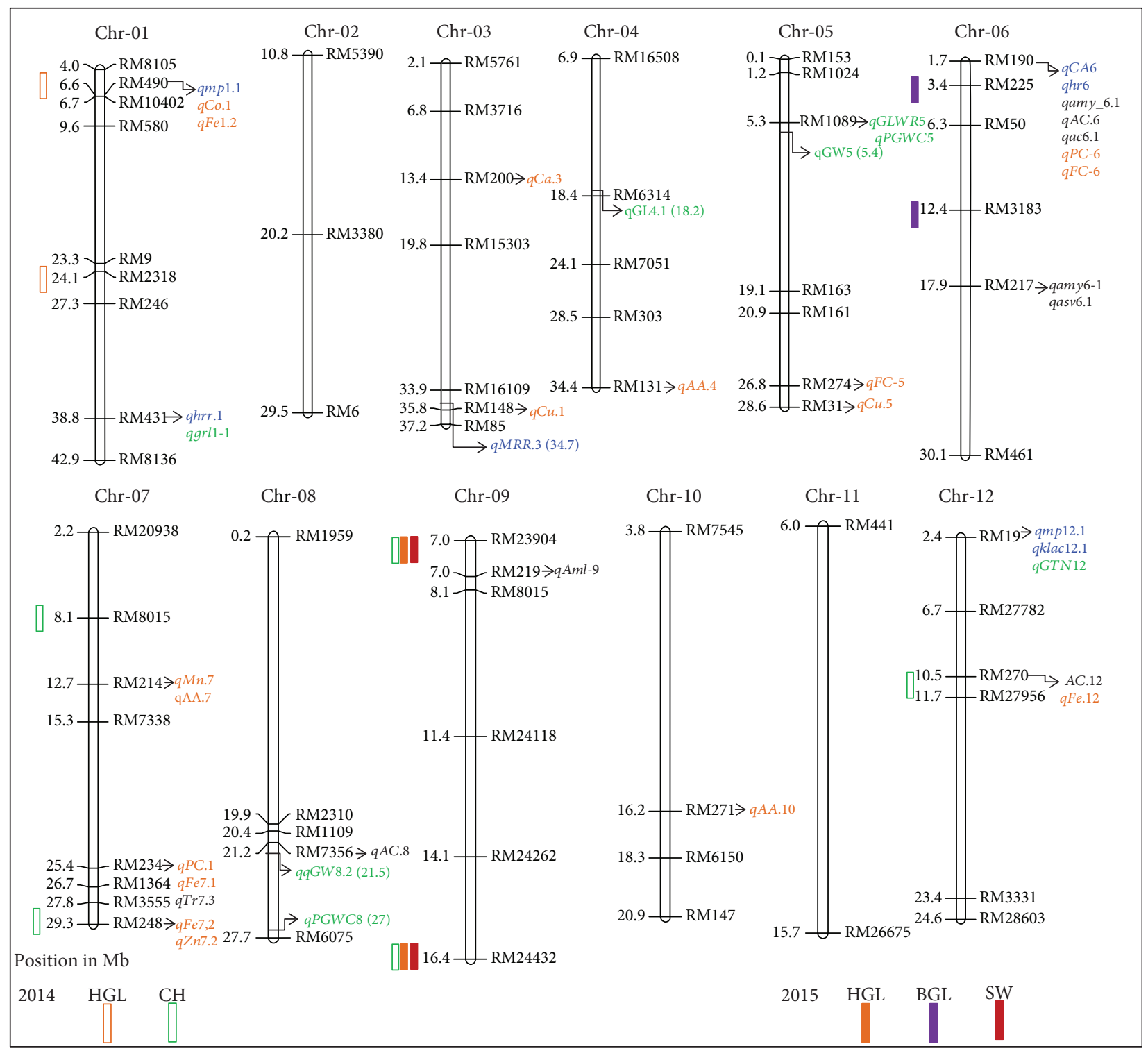

FIGURE 3: Distribution of QTLs for grain appearance and validation with previous reports on the linkage map. The hollow and solid bars indicate the QTLs detected in 2014 and 2015, respectively. The left and right sides indicate the polymorphic SSR marker physical positions $(\mathrm{Mb})$ associated with previous reports. A color font of each QTL represents the classification of grain appearance and nutritional quality traits. Blue color fonts are related to milling properties of grain quality (MPGQ) QTLs, green color fonts for grain appearance (GA) QTLs, orange color fonts for nutritional factor- (NF-) related QTL (NF), and black color font for cooking property (CP) QTLs in rice.

adjacent $5.4 \mathrm{Mb}$ regions responsible for grain length to width ratio (GLWR), the percentage of grain with chalkiness (PGWC) and grain width (GW), respectively. Two QTLs for fat content ( $q F C-5$, Yu et al. [58]) and microelement ( $q$ Cu.5, Lu et al. [56]) were associated with RM274 and RM31. Polymorphic markers to select for fat content and microelements can be highly useful for fair-sized rice breeding programs.

On chromosome 6, single QTL qBGL_6 (2015) were mapped at $52 \mathrm{cM}$ regions was flanked by RM225 and RM3183, which is associated with BGL, and it was explained by a PV of 6.70\%. This QTL was identified as a novel QTL and has not been previously reported. Near the RM225, polymorphic RM190 located at the $1.7 \mathrm{Mb}$ region which was significantly associated with seven QTLs, namely,
chalkiness-qCA6 [59], head rice recovery-qhr6 [14, 33], protein and fat content-qPC_6 and $q F C \_6$ [58], and three amylose content QTLs-qamy_6.1, qAC.6, and qac6.1 [14, 60, 61] were reported. These QTLs were identified with the genetic background of RILs derived from a cross between Xieqingzao B and Milyang [58], ZS97 and NYZ [59], Cypress and Panda [14], and another population of DHs derived from Caiapo and O. glaberrima [33]. Polymorphic markers like RM190 hold a high value and could be used directly in the marker-assisted selection of multiple traits.

The polymorphic markers RM214 $(12.7 \mathrm{Mb})$ associated with $q M n .7$ [36] and $q A A .7$ [57]; RM234 (25.3 Mb) for three QTLs, $q$ PC.1 [62], qFe7.1 [54], and $q \operatorname{Tr} 7.3$ [10]; and also adjacent to RM8015 (8.4 Mb) for single QTL qBRR7 [10] were responsible for various milling properties of grain quality 
and nutritional mineral element contents in rice grain. Four QTLs for three traits, CH, HGL, and SW, were detected by ICIM. These include one QTL $q C H \_1$ in 2014 and the remaining three QTLs $q H G L \_9, q S W \_9$, and $q S W \_9-1$ were identified in 2015, which explained 5.01\%, 7.22\%, 6.08\%, and $10.18 \%$, respectively. The clusters of QTLs were not reported by previous results of grain appearance and nutritional quality trait-related QTLs and markers. On chromosome 12, single QTL $q C H \_12$ for $\mathrm{CH}$ was identified by the ICIM method.

To date, more than 82 QTLs governing chalkinessrelated traits (http://archive.gramene.org/qtl/) including 30 QTLs for percentage grain with white core (PGWC), 26 QTLs for degree of endosperm chalkiness (DEC), 12 QTLs for area of endosperm chalkiness (AEC), 11 QTLs for white-backed kernel, and 3 QTLs for basal white (BW) were reported [28,63]. However, recently, two significant QTLs for chalkiness related as PGWC ( $q P G W C 6)$ and chalkiness score (CS) (qCS6) on chromosome 6 were reported by Chandusingh et al. [64]; 79 QTLs for six chalkiness-related traits as white core rate, white core area, white belly rate, white belly area chalkiness rate, and chalkiness area on 12 chromosomes by Peng et al. [59]; and also 19 QTLs for chalkiness on six different chromosomes, 1, 4, 6, 7, 9, and 12 , by Gao et al. [65] reported by using DHs and RIL populations. By comparison of previously reported QTLs and databases, qCH_12 flanked by right marker RM270 was associated with three nutritional-related QTLs such as $q A C .12$ [60] and $q F e .12$ [66] by using $\mathrm{DH}$ populations (Yuefu/IRAT109 and IR64/Azucena). The polymorphic markers RM219 on chromosome 9 ( $q A m l-9)$, RM271 on chromosome 10 (qAA.10), and RM19 on chromosome 12 (qmp12.1, qklac12.1, and qGTN12) were associated with MPGQ-, NF-, and GA-related traits [53, 57, 67]. In this study, we have validated the identified QTLs and polymorphic SSR markers based on published resources and databases. Several polymorphic markers and identified QTLs were associated with more than two traits and colocalized on chromosomes 1, 6, and 9, which could be utilized to improve the selection efficiency in rice breeding programs. The multiple traits on single locus may have multiple effects on each other as they belong to the identical genomic position. These could be due to a pleiotropic effect of a single gene or certain genes coexisting in the same QTLs. A further deeper understanding of fine mapping of the target genomic region would provide a genetic inheritance pattern and better picture to understand the linkage or pleiotropic effects on grain appearance and quality trait in rice.

\section{Conclusion}

Grain appearance and nutritional quality traits are vital for commercial rice production, and they also affect the dietary value of the grain. Considerable grain appearance traits in paddy, brown, and head rice were observed in the current study. Through ICIM-ADD methods, a total of seven QTLs for four traits, namely, HGL, BGL, SW, and $\mathrm{CH}$, were identified on four chromosomes, 1, 6, 9, and 12. By comparison of the comprehensive literature survey and publically accessible
Gramene database, 19 polymorphic markers were significantly associated with several traits related to MP, GA, CP, and NF. The four trait-associated QTLs are novel on chromosomes 6 and 9. Therefore, these QTLs and validated SSR polymorphic markers could provide valuable information for multiple traits related to grain appearance and nutritional quality for the future marker-assisted breeding program for improving desirable traits in rice.

\section{Data Availability}

All the relevant data has been incorporated into the original research manuscript.

\section{Conflicts of Interest}

The authors declare that the research review was conducted in the absence of any commercial or economic associations that could be construed as a potential conflict of interest.

\section{Authors' Contributions}

LBK, BAF, and MHC conducted a grain appearance phenotypic experiment. LBK, BAF, MHC, and NM performed the data collection and analysis. LBK, BAF, and AM were responsible for the analysis and interpretation of genotyping data. AM and LBK performed the QTL mapping and wrote the manuscript. AM and JA revised the manuscript. JA conceived the study, designed the experiments, gave suggestions, revised the entire article, and approved the manuscript. All authors read and approved the research article. Leila Bazrkar-Khatibani, Barat-Ali Fakheri, and Maryam Hosseini-Chaleshtori contributed equally to this work.

\section{Acknowledgments}

The authors would like to thank and acknowledge the Bill and Melinda Gates Foundation (BMGF) for providing a research grant to ZL for the Green Super Rice Project under ID OPP1130530. We also like to thank the Department of Science and Technology (DOST), Philippine Council for Agriculture, Aquatic, Natural Resources Research and Development (PCCARRD), Philippines, for providing funds to JA through the University of the Philippines Los Baños (UPLB) under the Improved Resource Use Efficient (iRUE) Rice Project.

\section{Supplementary Materials}

Supplementary Table 1: list of rice grain appearance and quality traits of associated QTLs in the different genetic background of mapping populations. Supplementary Table 2: a list of SSR markers has been used in the QTL mapping study. (Supplementary Materials)

\section{References}

[1] X. Kong, S. Kasapis, and J. Bao, "Viscoelastic properties of starches and flours from two novel rice mutants induced by gamma irradiation," LWT - Food Science and Technology, vol. 60 , no. 1, pp. 578-582, 2015. 
[2] D. S. Brar and G. S. Khush, "Wild relatives of rice: a valuable genetic resource for genomics and breeding research," in The Wild Oryza Genomes, Compendium of Plant Genomes, T. Mondal and R. Henry, Eds., pp. 1-25, Springer, Cham, 2018.

[3] N. Sreenivasulu, V. M. Butardo Jr, G. Misra, R. P. Cuevas, R. Anacleto, and P. B. Kavi Kishor, "Designing climate-resilient rice with ideal grain quality suited for high-temperature stress," Journal of Experimental Botany, vol. 66, no. 7, pp. 1737-1748, 2015.

[4] J. Sun, L. Yang, J. Wang et al., "Identification of a cold-tolerant locus in rice (Oryza sativa L.) using bulked segregant analysis with a next-generation sequencing strategy," Rice, vol. 11, no. 1, p. 24, 2018.

[5] Y. Amarawathi, R. Singh, A. K. Singh et al., "Mapping of quantitative trait loci for basmati quality traits in rice (Oryza sativa L.)," Molecular Breeding, vol. 21, no. 1, pp. 49-65, 2008.

[6] J. Bao, "Genes and QTLs for rice grain quality improvement," in Rice - Germplasm, Genetics and Improvement, InTech, 2014.

[7] B. O. Juliano and C. P. Villareal, Grain Quality Evaluation of World Rices, Int. Rice Res. Inst, 1993.

[8] A. Anandan, G. Rajiv, R. Eswaran, and M. Prakash, "Genotypic variation and relationships between quality traits and trace elements in traditional and improved rice (Oryza sativa L.) genotypes," Journal of Food Science, vol. 76, no. 4, pp. H122-H130, 2011.

[9] A. Mahender, A. Anandan, S. K. Pradhan, and E. Pandit, "Rice grain nutritional traits and their enhancement using relevant genes and QTLs through advanced approaches," Springerplus, vol. 5, no. 1, article 2086, 2016.

[10] X. Wang, Y. Pang, C. Wang et al., "New candidate genes affecting rice grain appearance and milling quality detected by genome-wide and gene-based association analyses," Frontiers in Plant Science, vol. 7, article 1998, 2017.

[11] R. P. Cuevas, V. O. Pede, J. McKinley, O. Velarde, and M. Demont, "Rice grain quality and consumer preferences: a case study of two rural towns in the Philippines," PLoS One, vol. 11, no. 3, article e $0150345,2016$.

[12] L. J. Unnevehr, B. O. Juliano, and C. M. Perez, "Consumer demand for rice grain quality in Southeast Asia," Rice Grain Quality Mark International Rice Research Institute, vol. 933, pp. 15-23, 1985.

[13] B. O. Juliano, Ed.B. O. Juliano, "Criteria and Tests for Rice Grain Qualities," in Rice Chemistry and Technology, B. O. Juliano, Ed., The American Association of Cereal Chemists, Inc., St. Paul, Minnesota, USA, 1985.

[14] J. L. Kepiro, A. M. McClung, M. H. Chen, K. M. Yeater, and R. G. Fjellstrom, "Mapping QTLs for milling yield and grain characteristics in a tropical japonica long grain cross," Journal of Cereal Science, vol. 48, no. 2, pp. 477-485, 2008.

[15] P. Roy, T. Orikasa, H. Okadome, N. Nakamura, and T. Shiina, "Processing conditions, rice properties, health and environment," International Journal of Environmental Research and Public Health, vol. 8, no. 6, pp. 1957-1976, 2011.

[16] H.-Y. Park, K.-W. Lee, and H.-D. Choi, "Rice bran constituents: immunomodulatory and therapeutic activities," Food \& Function, vol. 8, no. 3, pp. 935-943, 2017.

[17] C. Fabian and Y.-H. Ju, "A review on rice bran protein: its properties and extraction methods," Critical Reviews in Food Science and Nutrition, vol. 51, no. 9, pp. 816-827, 2011.
[18] P. Suwannaporn and A. Linnemann, "Rice-eating quality among consumers in different rice grain preference countries," Journal of Sensory Studies, vol. 23, no. 1, pp. 1-13, 2008.

[19] W. Sun, Q. Zhou, Y. Yao, X. Qiu, K. Xie, and S. Yu, "Identification of genomic regions and the isoamylase gene for reduced grain chalkiness in rice," PLoS One, vol. 10, no. 3, article e0122013, 2015.

[20] Y. F. Tan, Y. Z. Xing, J. X. Li, S. B. Yu, C. G. Xu, and Q. Zhang, "Genetic bases of appearance quality of rice grains in Shanyou 63, an elite rice hybrid," Theoretical and Applied Genetics, vol. 101, no. 5-6, pp. 823-829, 2000.

[21] J. Lou, L. Chen, G. Yue et al., "QTL mapping of grain quality traits in rice," Journal of Cereal Science, vol. 50, no. 2, pp. 145-151, 2009.

[22] A. Anandan, T. Sabesan, R. Eswaran, G. Rajiv, N. Muthalagan, and R. Suresh, "Appraisal of environmental interaction on quality traits of rice by additive main effects and multiplicative interaction analysis," Cereal Research Communications, vol. 37, no. 1, pp. 131-140, 2009.

[23] C. Yin, H. Li, S. Li, L. Xu, Z. Zhao, and J. Wang, "Genetic dissection on rice grain shape by the two-dimensional image analysis in one japonica $\times$ indica population consisting of recombinant inbred lines," Theoretical and Applied Genetics, vol. 128, no. 10, pp. 1969-1986, 2015.

[24] C. Yin, H. Li, Z. Zhao et al., "Genetic dissection of top three leaf traits in rice using progenies from a japonica $\times$ indica cross," Journal of Integrative Plant Biology, vol. 59, no. 12, pp. 866880, 2017.

[25] P.-R. Yuan, H.-J. Kim, Q.-H. Chen, H.-G. Ju, S.-D. Ji, and S.-N. Ahn, "Mapping QTLs for grain quality using an introgression line population from a cross between Oryza sativa and O. rufipogon," Journal of Crop Science and Biotechnology, vol. 13, no. 4, pp. 205-212, 2010.

[26] J. C. Nelson, F. Jodari, A. I. Roughton et al., "QTL mapping for milling quality in elite western U.S. rice germplasm," Crop Science, vol. 52, no. 1, pp. 242-252, 2012.

[27] A. Zhu, Y. Zhang, Z. Zhang et al., "Genetic dissection of $q P C G 1$ for a quantitative trait locus for percentage of chalky grain in rice (Oryza sativa L.)," Frontiers in Plant Science, vol. 9, article 1173, 2018.

[28] L. I. ZeOFu, W. A. N. JianOMin, X. I. A. JiaOFa, and Z. H. A. I. $\mathrm{HuOQu}$, "Mapping quantitative trait loci underlying appearance quality of rice grains (Oryza sativa L.),”遗传学报, vol. 30, no. 3, 2003.

[29] X. Y. Wan, J. M. Wan, J. F. Weng et al., "Stability of QTLs for rice grain dimension and endosperm chalkiness characteristics across eight environments," Theoretical and Applied Genetics, vol. 110, no. 7, pp. 1334-1346, 2005.

[30] E. D. Redona and D. J. Mackill, "Quantitative trait locus analysis for rice panicle and grain characteristics," Theoretical and Applied Genetics, vol. 96, no. 6-7, pp. 957-963, 1998.

[31] Y. Zhang, Y. H. Zhang, S. L. Dong et al., "QTL mapping for grain size traits based on extra-large grain rice line TD70," Rice Science, vol. 20, no. 6, pp. 400-406, 2013.

[32] B. Rabiei, M. Valizadeh, B. Ghareyazie, M. Moghaddam, and A. J. Ali, "Identification of QTLs for rice grain size and shape of Iranian cultivars using SSR markers," Euphytica, vol. 137, no. 3, pp. 325-332, 2004.

[33] G. Aluko, C. Martinez, J. Tohme, C. Castano, C. Bergman, and J. H. Oard, "QTL mapping of grain quality traits from the interspecific cross Oryza sativa $\times$ O. glaberrima," 
Theoretical and Applied Genetics, vol. 109, no. 3, pp. 630639, 2004.

[34] C. Fan, Y. Xing, H. Mao et al., "GS3, a major QTL for grain length and weight and minor QTL for grain width and thickness in rice, encodes a putative transmembrane protein," Theoretical and Applied Genetics, vol. 112, no. 6, pp. 1164-1171, 2006.

[35] T. Q. Zheng, J. L. Xu, Z. K. Li, H. Q. Zhai, and J. M. Wan, "Genomic regions associated with milling quality and grain shape identified in a set of random introgression lines of rice (Oryza sativa L.)," Plant Breeding, vol. 126, no. 2, pp. 158163, 2007.

[36] M. Zhang, S. R. M. Pinson, L. Tarpley et al., "Mapping and validation of quantitative trait loci associated with concentrations of 16 elements in unmilled rice grain," Theoretical and Applied Genetics, vol. 127, no. 1, pp. 137-165, 2014.

[37] X. Wan, J. Weng, H. Zhai et al., "Quantitative trait loci (QTL) analysis for rice grain width and fine mapping of an identified QTL allele gw-5 in a recombination hotspot region on chromosome 5," Genetics, vol. 179, no. 4, pp. 2239-2252, 2008.

[38] X. Bai, L. Luo, W. Yan, M. R. Kovi, W. Zhan, and Y. Xing, "Genetic dissection of rice grain shape using a recombinant inbred line population derived from two contrasting parents and fine mapping a pleiotropic quantitative trait locus qGL7," BMC Genetics, vol. 11, no. 1, p. 16, 2010.

[39] G. Shao, S. Tang, J. Luo et al., "Mapping of qGL7-2, a grain length QTL on chromosome 7 of rice," Journal of Genetics and Genomics, vol. 37, no. 8, pp. 523-531, 2010.

[40] Q. Dong, Z. H. Zhang, L. L. Wang et al., "Dissection and fine-mapping of two QTL for grain size linked in a $460-\mathrm{kb}$ region on chromosome 1 of rice," Rice, vol. 11, no. 1, p. 44, 2018.

[41] N. Li and Y. Li, "Signaling pathways of seed size control in plants," Current Opinion in Plant Biology, vol. 33, pp. 23-32, 2016.

[42] N. Tabkhkar, B. Rabiei, and A. Sabouri, "Genetic diversity of rice cultivars by microsatellite markers tightly linked to cooking and eating quality," Australian Journal of Crop Science, vol. 6, no. 6, p. 980, 2012.

[43] M. Janwan, T. Sreewongch, and P. Sripichitt, "Rice breeding for high yield by advanced single seed descent method of selection," Journal of Plant Sciences, vol. 8, no. 1, pp. 24-30, 2013.

[44] M. G. Murray and W. F. Thompson, "Rapid isolation of high molecular weight plant DNA," Nucleic Acids Research, vol. 8, no. 19, pp. 4321-4326, 1980.

[45] S. Temnykh, G. DeClerck, A. Lukashova, L. Lipovich, S. Cartinhour, and S. McCouch, "Computational and experimental analysis of microsatellites in rice (Oryza sativa L.): frequency, length variation, transposon associations, and genetic marker potential," Genome Research, vol. 11, no. 8, pp. 14411452, 2001.

[46] S. R. McCouch, L. Teytelman, Y. Xu et al., "Development and mapping of 2240 new SSR markers for rice (Oryza sativa L.)," DNA Research, vol. 9, no. 6, pp. 199-207, 2002.

[47] S. Creste, A. T. Neto, and A. Figueira, "Detection of single sequence repeat polymorphisms in denaturing polyacrylamide sequencing gels by silver staining," Plant Molecular Biology Reporter, vol. 19, no. 4, pp. 299-306, 2001.

[48] B. J. Bassam and G. Caetano-Anollés, "Silver staining of DNA in polyacrylamide gels," Applied Biochemistry and Biotechnology, vol. 42, no. 2-3, pp. 181-188, 1993.
[49] L. Meng, H. Li, L. Zhang, and J. Wang, "QTL IciMapping: integrated software for genetic linkage map construction and quantitative trait locus mapping in biparental populations," The Crop Journal, vol. 3, no. 3, pp. 269-283, 2015.

[50] H. Li, G. Ye, and J. Wang, "A modified algorithm for the improvement of composite interval mapping," Genetics, vol. 175, no. 1, pp. 361-374, 2007.

[51] W. M. Edzesi, X. Dang, L. Liang, E. Liu, I. U. Zaid, and D. Hong, "Genetic diversity and elite allele mining for grain traits in rice (Oryza sativa L.) by association mapping," Frontiers in Plant Science, vol. 7, p. 787, 2016.

[52] J. Nan, X. Feng, C. Wang et al., "Improving rice grain length through updating the GS3 locus of an elite variety Kongyu 131," Rice, vol. 11, no. 1, p. 21, 2018.

[53] B. P. M. Swamy, K. Kaladhar, N. Shobha Rani et al., "QTL analysis for grain quality traits in $2 \mathrm{BC} 2 \mathrm{~F} 2$ populations derived from crosses between Oryza sativa cv Swarna and 2 accessions of O. nivara," Journal of Heredity, vol. 103, no. 3, pp. 442-452, 2012.

[54] K. Anuradha, S. Agarwal, Y. V. Rao, K. V. Rao, B. C. Viraktamath, and N. Sarla, "Mapping QTLs and candidate genes for iron and zinc concentrations in unpolished rice of Madhukar× Swarna RILs," Gene, vol. 508, no. 2, pp. 233-240, 2012.

[55] E. M. Septiningsih, K. R. Trijatmiko, S. Moeljopawiro, and S. R. McCouch, "Identification of quantitative trait loci for grain quality in an advanced backcross population derived from the Oryza sativa variety IR64 and the wild relative O. rufipogon," Theoretical and Applied Genetics, vol. 107, no. 8, pp. 1433-1441, 2003.

[56] K. Lu, L. Li, X. Zheng, Z. Zhang, T. Mou, and Z. Hu, "Quantitative trait loci controlling $\mathrm{Cu}, \mathrm{Ca}, \mathrm{Zn}, \mathrm{Mn}$ and $\mathrm{Fe}$ content in rice grains," Journal of Genetics, vol. 87, no. 3, pp. 305-310, 2008.

[57] L. Wang, M. Zhong, X. Li et al., "The QTL controlling amino acid content in grains of rice (Oryza sativa) are co-localized with the regions involved in the amino acid metabolism pathway," Molecular Breeding, vol. 21, no. 1, pp. 127-137, 2008.

[58] Y.-H. Yu, G. Li, Y. Y. Fan et al., "Genetic relationship between grain yield and the contents of protein and fat in a recombinant inbred population of rice," Journal of Cereal Science, vol. 50, no. 1, pp. 121-125, 2009.

[59] B. Peng, L. Wang, C. Fan et al., "Comparative mapping of chalkiness components in rice using five populations across two environments," BMC Genetics, vol. 15, no. 1, p. 49, 2014.

[60] C. C. Fan, X. Q. Yu, Y. Z. Xing, C. G. Xu, L. J. Luo, and Q. Zhang, "The main effects, epistatic effects and environmental interactions of QTLs on the cooking and eating quality of rice in a doubled-haploid line population," Theoretical and Applied Genetics, vol. 110, no. 8, pp. 1445-1452, 2005.

[61] J. C. Nelson, A. M. McClung, R. G. Fjellstrom et al., "Mapping QTL main and interaction influences on milling quality in elite US rice germplasm," Theoretical and Applied Genetics, vol. 122, no. 2, pp. 291-309, 2011.

[62] Y. F. Tan, M. Sun, Y. Z. Xing et al., "Mapping quantitative trait loci for milling quality, protein content and color characteristics of rice using a recombinant inbred line population derived from an elite rice hybrid," Theoretical and Applied Genetics, vol. 103, no. 6-7, pp. 1037-1045, 2001.

[63] A. Kobayashi, B. Genliang, Y. Shenghai, and K. Tomita, "Detection of quantitative trait loci for white-back and 
basal-white kernels under high temperature stress in japonica rice varieties," Breeding Science, vol. 57, no. 2, pp. 107-116, 2007.

[64] P. R. Chandusingh, N. K. Singh, K. V. Prabhu, K. K. Vinod, and A. K. Singh, "Molecular mapping of quantitative trait loci for grain chalkiness in rice (Oryza sativa L.)," Indian Journal of Genetics and Plant Breeding (The), vol. 73, no. 3, pp. 244-251, 2013.

[65] X. Gao, X. Zhang, H. Lan, J. Huang, J. Wang, and H. Zhang, "The additive effects of GS3 and $q G L 3$ on rice grain length regulation revealed by genetic and transcriptome comparisons," BMC Plant Biology, vol. 15, no. 1, p. 156, 2015.

[66] J. C. R. Stangoulis, B. L. Huynh, R. M. Welch, E. Y. Choi, and R. D. Graham, "Quantitative trait loci for phytate in rice grain and their relationship with grain micronutrient content," Euphytica, vol. 154, no. 3, pp. 289-294, 2007.

[67] B.-W. Yun, M. G. Kim, T. Handoyo, and K. M. Kim, “Analysis of rice grain quality-associated quantitative trait loci by using genetic mapping," American Journal of Plant Sciences, vol. 5, no. 9, pp. 1125-1132, 2014. 


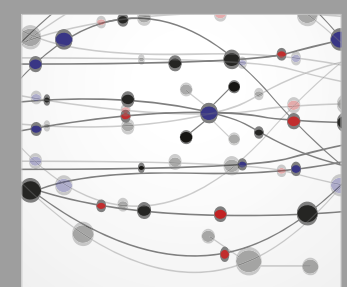

The Scientific World Journal
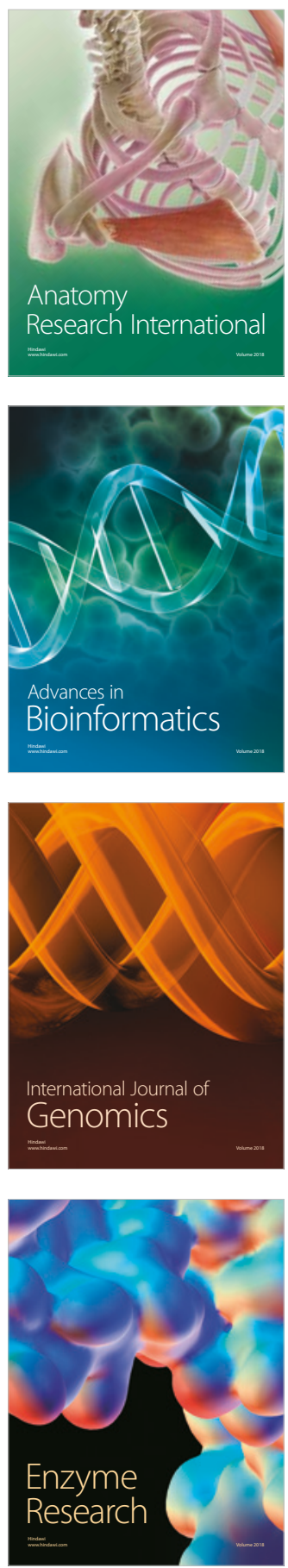
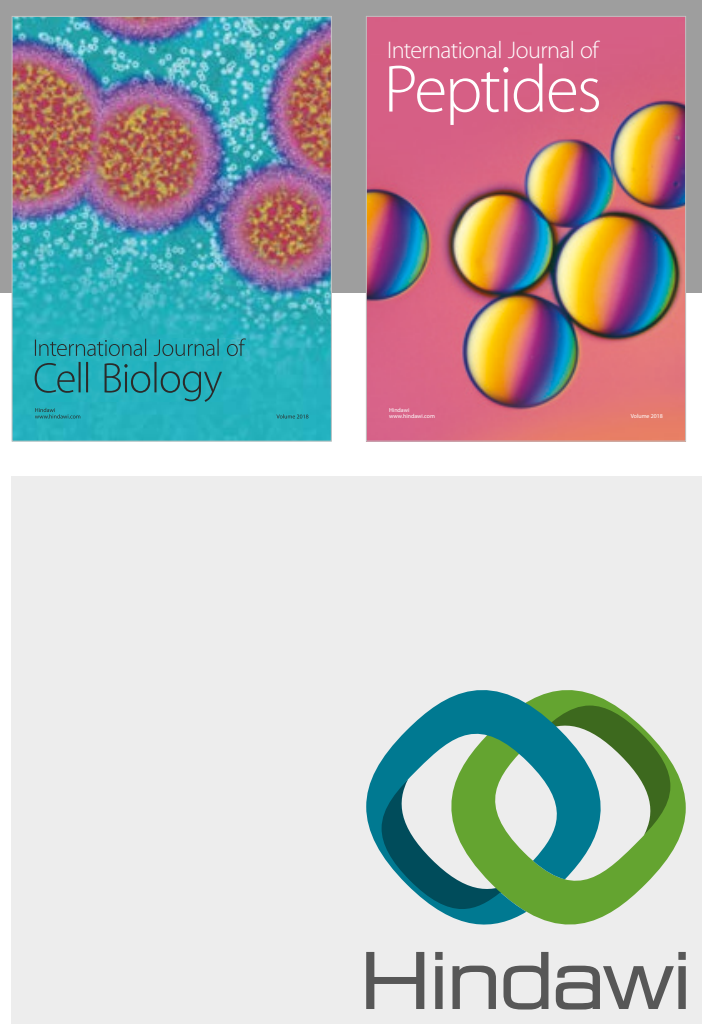

Submit your manuscripts at

www.hindawi.com
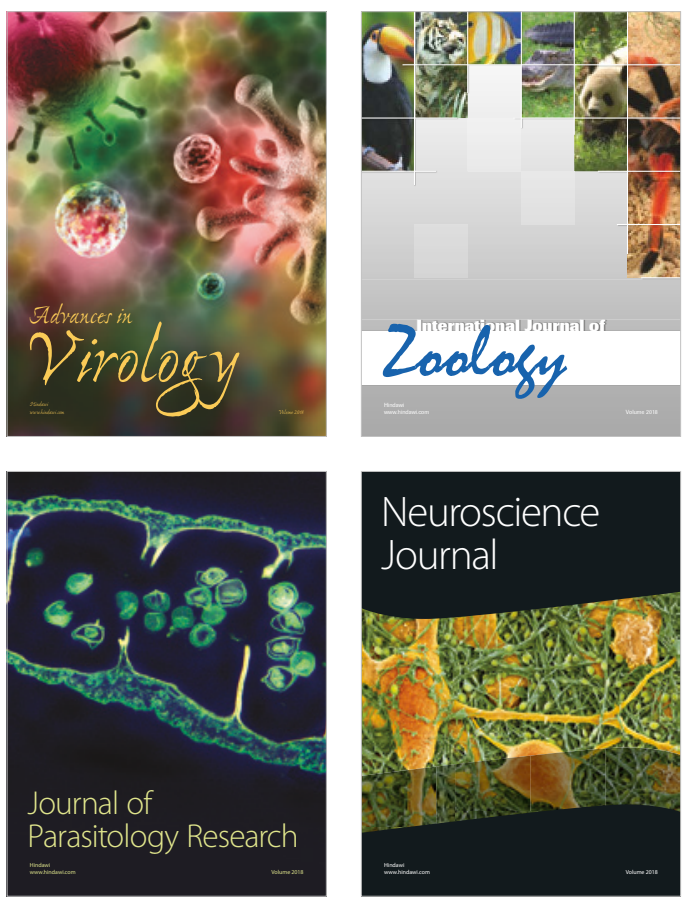
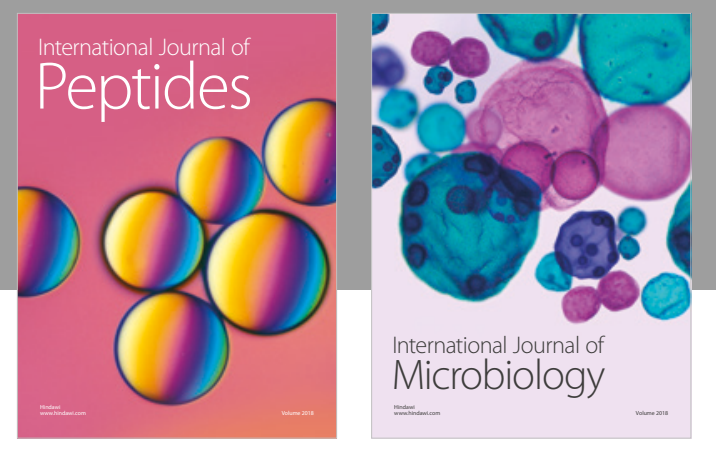

nternational Journal of Microbiology
Journal of
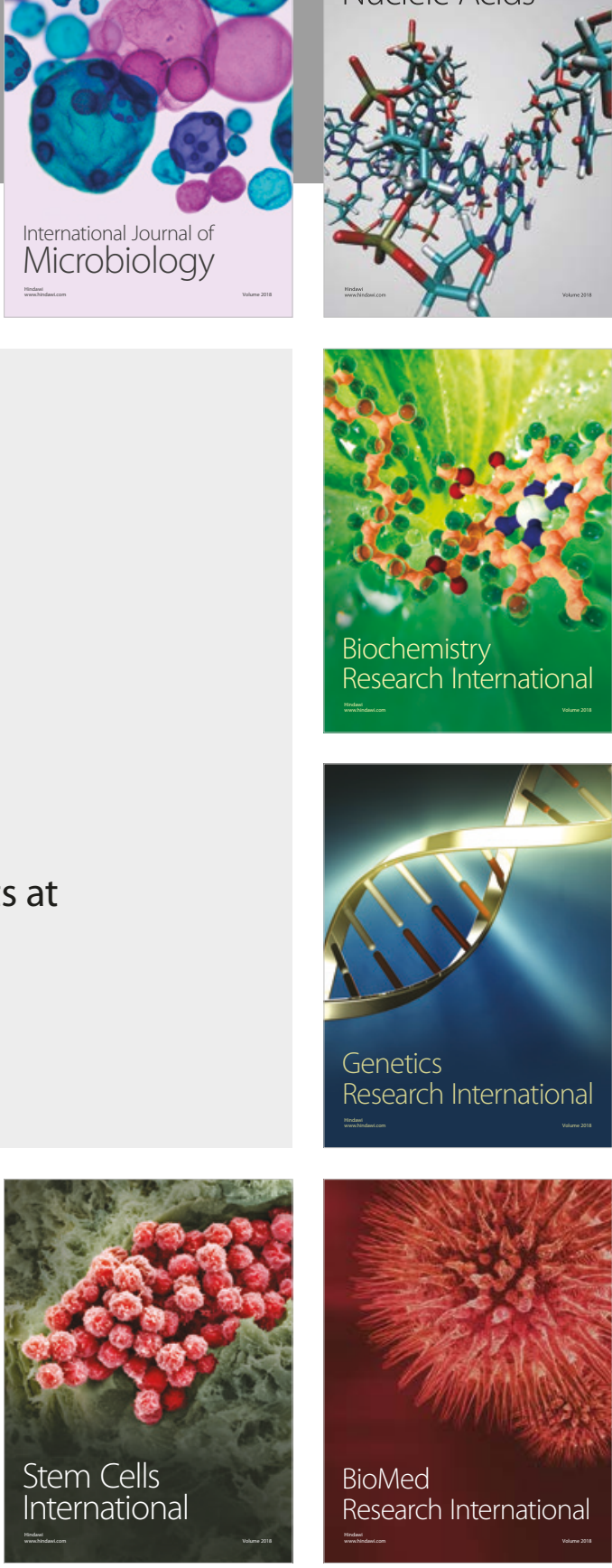
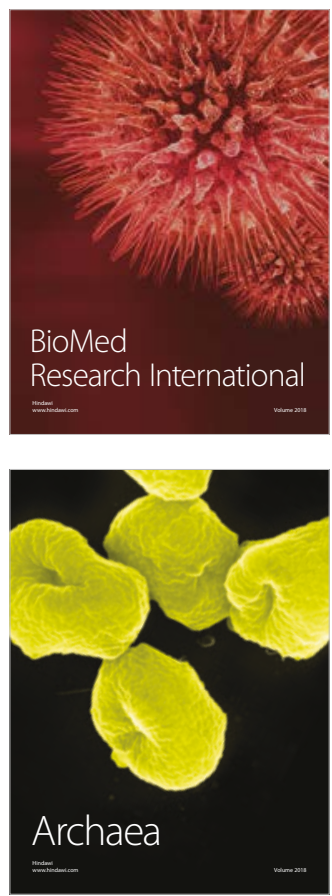